PERSONAL PRACTICE

\title{
Botulinum toxin for cerebral palsy; where are we now?
}

\author{
R E Morton, J Hankinson, J Nicholson
}

Arch Dis Child 2004;89:1133-1137. doi: 10.1136/adc.2003.044560

In this article, the evidence base for botulinum-A treatment acquired in recent years is outlined, and the practicalities involved in providing this service are described. Botulinum$A$ is relatively new, and possible improvements for the future are considered.

See end of article for authors' affiliations .....................

Correspondence to: Dr R E Morton, Ronnie MacKeith Child Development Centre, Derbyshire Children's' Hospital, Derby and Nottingham Queens Medical Centre and City Hospital, Nottingham, UK; richard.morton@sdah-tr. trent.nhs.uk

Accepted 4 April 2004
B otulinum-A (BT-A) injections were first given therapeutically for strabismus in the early 1980s by Alan Scott in the USA. ${ }^{1}$ The treatment was adopted for other conditions and reports of its use in spastic muscle in cerebral palsy $(\mathrm{CP})$ began to appear a decade later. ${ }^{23}$ Since then it has become a standard treatment for hypertonus in individual muscles in $\mathrm{CP}$, as an alternative to global therapy such as oral or intrathecal baclofen. When injected into muscle, BT-A is tightly bound to motor end plates where it impairs the release of acetylcholine, causing a chemical denervation lasting 3-4 months. Full recovery then occurs, not, as was once thought by alternative nerve sprouting, but probably by gradual degradation of either the abnormal "snare" protein produced by the toxin, or the toxin itself. ${ }^{4}$

BT-A will therefore only work on hypertonus can be either spasticity, in which the electrical activity is switched on by muscle stretch, or dystonia, when continuous muscle activity fixes the limb in abnormal positions. It has no effect on hypertonus caused by biomechanical changes in muscle, such as plasticity or thixotropy, ${ }^{5}$ or once significant contracture (that is, muscle shortening) has developed. ${ }^{6}$

Physiotherapists and occupational therapists are pivotal to the management of CP along with medical and surgical colleagues, and should be involved in careful assessment before and after the injections. This treatment is safe and effective and could now be offered at district level as part of an overall package of care for $\mathrm{CP}$, with occasional help from regional centres for more complex cases.

\section{EVIDENCE OF EFFECT}

Randomised controlled trials (RCTs) provide the most reliable evidence of benefit of new treatments such as BT-A. ${ }^{7}$ Several have now been conducted on its use in the lower limb, mainly looking at gait changes following injections to the calf muscle. These have been shown to be better than placebo injections using a variety of measures: video recordings of gait analysed with the Physician Rating Scale, ${ }^{89}$ laboratory gait analysis, $^{10}$ and the Gross Motor Function Measure. ${ }^{11}$ Two RCTs have compared injections caused by electrical overactivity in muscle. This with below knee casting, one showing BT-A to be better, $^{12}$ and the other the same, ${ }^{13}$ although a combined meta-analysis confirmed the superiority of BT-A. ${ }^{14}$ Injections to the hip adductors peri-operatively have been shown to reduce pain in an RCT. ${ }^{15}$ There have also been many open label studies showing the benefit of BT-A injections to different leg muscles. ${ }^{14}$

By contrast, there has been relatively little research into the use of BT-A in the upper limbs in CP; only two full RCTs have compared BT-A with placebo injections. One found that tone is reduced, with improvement in cosmesis and some upper limb functions. It also showed that the weakness induced may interfere with pinch grip, and concluded that the outcome varied considerably between subjects. ${ }^{16}$ The other showed the benefit of injections for active function in hemiplegics using the QUEST scales. ${ }^{17}$ A later analysis of this study could not show a clear relation between QUEST total score and the benefit obtained, except that grip strength improved more in those with a reasonable grip to start with. ${ }^{18}$ Using movement analysis, we have found that BT-A injections to elbow flexors can actually inhibit usage of these joints in some subjects, ${ }^{19}$ emphasising the importance of careful patient selection and later assessment for upper limb injections. A beneficial outcome is more certain if the injections are intended to improve cosmesis, or passive functioning for care giving in dressing, washing, etc. ${ }^{20} 21$

There has therefore been considerable progress since the initial descriptions of BT-A treatment in $\mathrm{CP}^{22}$ and suggestions that any benefit remained unproven, ${ }^{23}$ although more research is still needed in particular areas. For example, it is not yet clear whether repeated injections maintain their effect, or reduce contracture formation as shown in spastic mice. ${ }^{24}$ In a small series of 11 children receiving treatment to the calf muscle over three years, we found a slight diminution in response in some individuals, probably due to the development of contractures; two needed surgical elongation. ${ }^{25}$ Antibodies against BT-A can occur with long term treatment, given the large amounts of toxin administered, but most are probably insignificant as they affect inactive sites of the BT-A complex. ${ }^{26}$ Nevertheless, some feel this may account for non-response in up to $15 \%$ subjects. $^{27}$

Abbreviations: BT-A, botulinum A; CP, cerebral palsy; $R C T$, randomised controlled trial 


\section{PRACTICALITIES OF TREATMENT Assessment}

Assessment and planning for this treatment needs to be shared between the multidisciplinary team. Before injection, clear objectives must be set which are both achievable and of practical value. These are combined with assessments of movement, joint ranges, and muscle tone using the modified Ashworth scales. ${ }^{28}$ Repeat assessment is carried out after a couple of months to see if the goals have been achieved, and to plan further injections and therapy. A newly emerging skill may be affected-positively or negatively—and timing of the next injection may be crucial. During courses of long term treatment, these assessments need to be repeated every so often to adjust therapy.

For assessments of movement in the lower limb, we take a video recording in the saggital and coronal planes, slowing down the recording to score joint positions at different stages of the gait cycle using the lower limb Physicians Rating Scale. ${ }^{29}$ Sometimes full gait analysis is helpful to provide a more detailed picture in all three planes, especially for complex diplegics. For the upper limb, the equivalent Physicians Rating Scale can be used, ${ }^{30}$ often from direct observation without the need for a video. Sometimes more complex assessment of function is indicated, when one can use scales such as the Melbourne assessment of upper limb function, ${ }^{31}$ or the QUEST. ${ }^{32}$ Treatment to the upper limbs can take longer to have an effect in view of the complex motor adjustments involved, and assessments are best left until around four months. ${ }^{1620}$ We have tried using movement analysis of the upper limbs, but this takes considerable time with some children who may find the repetitive tasks involved difficult. ${ }^{19}$

A decision needs to be made on whether to continue with repeat injections. This can be difficult for the injector, with a degree of vested interest, or even the carer, who may be influenced by the placebo effect. One study comparing BT-A with placebo found that $26 \%$ of carers felt saline injections had been beneficial. ${ }^{33}$ We find that often the child's therapist can provide the objectivity required, taking the assessments and opinions of all concerned into consideration.

\section{Procedure for injection}

Young children respond best to treatment, from as early as 2 years of age. ${ }^{34}$ This is because they rarely have contractures and are more receptive to changes in motor patterning. BT-A has more effect if followed by therapy to stretch the muscle and revise movements. Carers can be asked to do this, supervised by the therapist who may arrange a package of

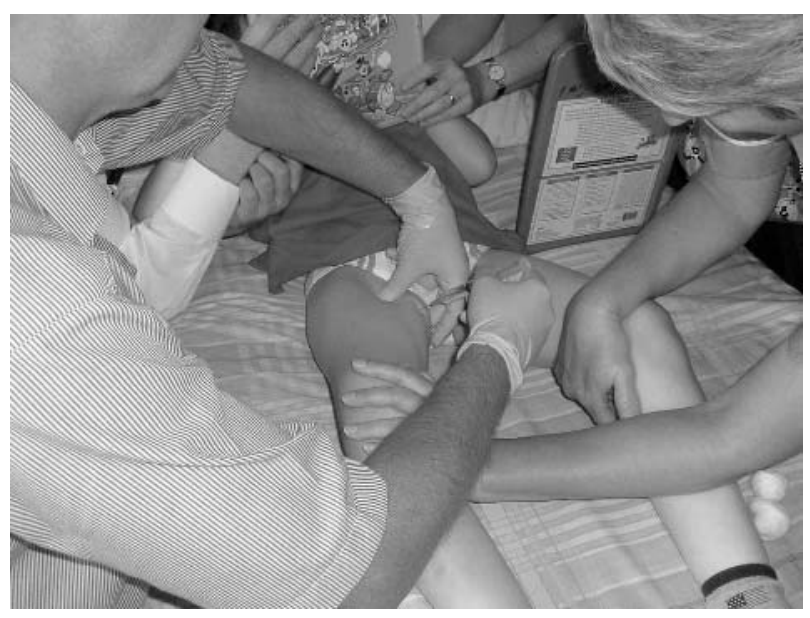

Figure 1 BT-A injection to the lower limbs. therapy to concentrate on the post-injection period. This can be achieved without increase in the overall therapist time involved. Post-injection splinting is also useful; for example, ankle foot orthoses to be used at night as well as by day, or elbow and wrist splints for the upper limb. For moderate ankle contractures allowing dorsiflexion to the neutral position, serial below knee plasters can be more effective if the calf muscle is first relaxed with BT-A. These are fitted one week after injection and changed two weekly on two or three occasions. ${ }^{12}$

BT-A is available as two preparations which are equal in effect and cost, but have different strengths, the equivalence ratio being around 1:3 (Botox BT-A (Allergan) : Dysport BT-A (Ipsen)). Each centre should only stock one preparation to avoid confusion. We use Dysport BT-A at a concentration of 100 units $/ \mathrm{ml}$, sometimes with increased concentration of 250 units/ml in small muscles of the upper limb. Conventional dosage schemes for treatment to different muscles are used in terms of units $/ \mathrm{kg}$, at sites determined by surface anatomy. We use an electrical muscle stimulator for deep muscles such as postrior tibialis, or small muscles difficult to localise in the forearm. ${ }^{35}$ Recommendations for maximal total dosage are 30 units/kg Dysport ${ }^{36}$ or 12 units/kg Botox; ${ }^{37}$ keeping to these guidelines, systemic side effects are extremely rare. These include mild generalised weakness, urinary incontinence, constipation, or dysphagia in vulnerable children; they start 7-10 days after the injections and may last a few days. Injections are given at 4-6 monthly intervals according to need and can be continued during the growing years. We have been successfully treating some children in this way for over seven years. Suggestions of how to run a botulinum service are summarised in box 1 , including multidisciplinary assessments, and arrangements for the injections themselves. We usually inject six children in one outpatient session, with additional general anaesthesia lists for psoas injections and when multiple injections are needed. Orthopaedic surgeons give the injections in many centres, but general paediatricians can also do this satisfactorily with appropriate training. Good nursing support is essential; at least two senior nurses together with other staff are required to support the carers and distract the child during the injections.

\section{Which muscles to treat}

Children with hypertonic $\mathrm{CP}$ are now grouped according to whether unilateral or bilateral, and severity on the Gross Motor Function Classification Score (GMFCS).$^{38}$ Indications for treatment with BT-A are determined by the type of CP and according to individual variation, which is considerable.

\section{Hemiplegia (unilateral CP, GMFCS 1, 2)}

Injection into gastrocnemius, often with soleus, has been shown extensively to improve ankle dorsiflexion in the stance and swing phases of gait. ${ }^{10}$ Sometimes the heel is in a varus position with an inverted mid-foot due to overactive tibialis posterior; this muscle can also be injected. These treatments maintain a better foot position in an ankle foot orthosis and can be used in conjunction with serial plastering if necessary. ${ }^{12}$ In more severely affected hemiplegics with hamstring spasticity, ${ }^{39}$ BT-A injection into this muscle is also helpful.

Injections to the upper limb can sometimes improve function in children with hemiplegia. Increased reach can be achieved with treatment to elbow flexors (biceps, brachialis, and brachio-radialis) and wrist flexors (flexor carpi radialis and ulnaris). Pronator teres may be included to improve supination, although this muscle often has a fixed contracture. Injections to the finger flexors (flexor digitorum sublimis and profundus) can be helpful, especially if release is more impaired than grasp..$^{40}$ The thumb in palm deformity 


\section{Box 1: Suggested protocol for botulinum service}

\section{Assessment}

- With physiotherapist/occupational therapist lor at least a written report from them)

- Set goals for injections; e.g. better elbow extension for keyboard

- Baseline assessment; video with Physician Rating Scale for lower limb, or the Upper Limb Physician Rating Scale (video unnecessary)

- Provide written information for parents (they may like to meet parents of a child who has had it done previously)

- Write up medications and indicate in notes where local anaesthetic needs to be applied to help nurses

Injection

- Nurse check to see they are well (don't inject if pyrexial) and check weight

- One hour before apply local anaesthetic. Mark the limb to be injected

- Doctor to obtain written consent

- Half an hour before give midazolam orally (parenteral preparation); $0.5 \mathrm{mg} / \mathrm{kg}$ up to $25 \mathrm{mg}$. Oral/ rectal diclofenac can also be used if needed. Inhaled nitrous oxide in addition to midazolam can be used for children with a developmental age of 6 or above for additional anxiolysis and analgesia

- During injection need one nurse for assistance and one for distraction (play therapists are excellent for this if available)

- Home half an hour after injections

- Report to GP, parents, therapists, etc

- Review one week post-injection by therapist to plan input, including preparation of splints for the upper limb. Serial below knee plastering can be started at this time if indicated

Review (2-3 months lower limb, 4 months upper limb)

- With therapists if possible, or at least a report from them

- Have the designated goals been achieved? What do parents and child think?

- Review assessment as before injections

- What changes next time-different doses, different muscles, more sedation?

- Only plan re-injection if clearly beneficial

- Re-injection every 4-6 months, with full repeat reassessment as necessary

is troublesome and relieved with injections to the flexor pollicis brevis and opponens pollicis.

\section{Diplegia (bilateral CP, GMFCS 2, 3, 4)}

Careful assessment is needed to determine those leg muscles responsible for gait deviations in mobile children with bilateral CP, and which may be suitable for injection. Crouch gait is common, often due to hip flexion from overactive psoas muscles. Either a posterior or anterior approach is used to this muscle, but both are difficult. The posterior approach from the back must be done under general anaesthesia with $x$ ray screening to ensure the peritoneum is

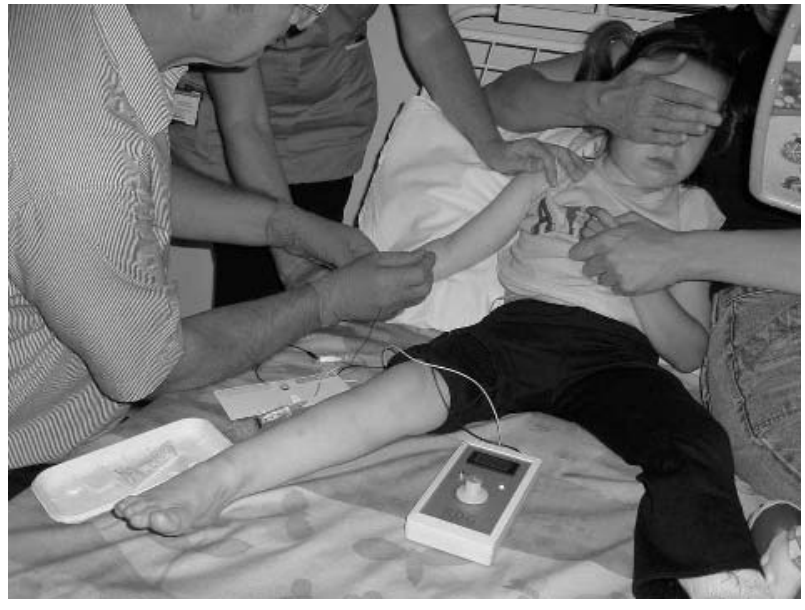

Figure 2 Injection of BT-A in the upper limbs using nerve stimulator.

not breached. ${ }^{41}$ Recently we have also used an anterior method in the groin, which can be done using ultrasound guidance and without general anaesthesia in a cooperative child. $^{42}$ Hip flexion produces knee flexion because the hamstrings act as both hip extensors and knee flexors ("hamstring shift"), so treatment to psoas also straightens the knees. There may be additional knee flexion from spasticity in the hamstrings which are easily injectable. Excessive knee flexion in stance is usually the main cause of toe walking in diplegics, together with a degree of calf muscle spasticity/contracture causing plantarflexion. The gastrocnemius can be injected, but care must be taken injecting the soleus as over-treatment can lead to collapse from uncontrolled dorsiflexion. Sometimes the foot is inverted and tibialis posterior injections are indicated. A Trendelenburg gait (dropping of the hip on the swing side), often combined with scissoring, is a possible indication for injections to the hip adductors. However these muscles are important for trunk progression in walking, and if injected mistakenly, the gait will deteriorate. Gait analysis in the coronal plane can be helpful in this situation, showing the swing leg hanging from a dropped pelvis. If the adductor is overactive on this side, it pulls the leg across the mid-line, but this does not happen if the pelvis drops due to opposite abductor weakness alone. BT-A can also be used in the situations described when there are significant muscle contractures in addition to spasticity, as a "dummy run" to plan surgery. ${ }^{43}$

Sometimes spasticity is widespread and focal injections with BT-A are insufficient, so other treatments have to be considered.. Oral antispasticity treatment, including baclofen, dantrolene, and tizanidine can be tried, but they are significantly limited by sedative side effects. Increasingly intrathecal baclofen from a pump is used for subjects with generalised very high tone, but further research is needed to determine which subjects benefit most, particularly if mobility is to be improved. ${ }^{44}$ In some countries dorsal rhizotomy is popular for reducing generalised hypertonia in the lower limbs, but this is largely out of favour in the UK. There are concerns that it is an invasive means of achieving only small functional gains, with potential significant complications, ${ }^{45}$ although this remains under review. ${ }^{43}$ Motor progress in diplegics slows down from around the age of 7 years $^{46}$ due to contracture formation and an increasing mismatch between weight and strength. By reducing contractures, anti-spasticity treatments including BT-A, may delay the need for multi-level surgery in these children, and hopefully may restrict the number of procedures eventually required. 


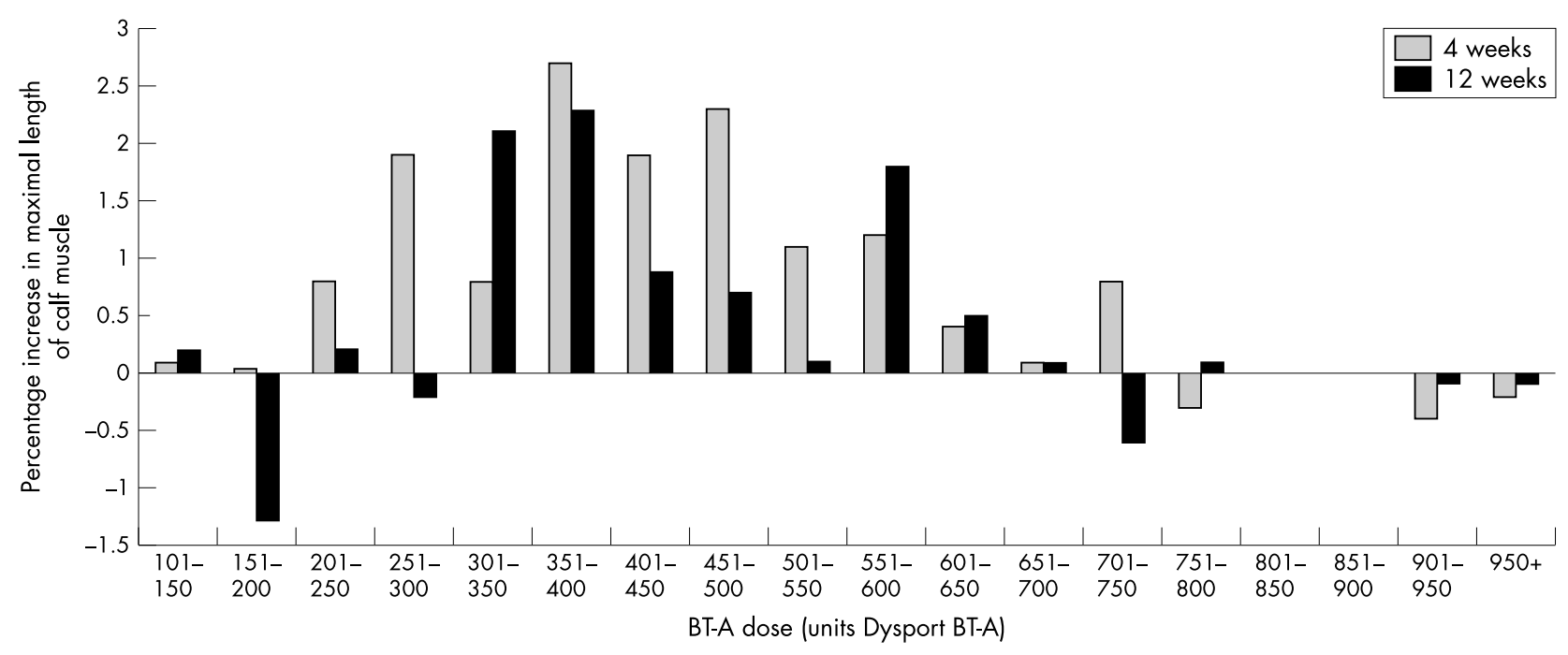

Figure 3 Response of calf muscle to BT-A expressed as absolute dosage.

Quadriplegia (bilateral CP, GMFCS 4, 5)

The most important goal of treatment of hypertonus in this group is the reduction of secondary soft tissue bony deformity, especially in the hips and spine. Children with bilateral $\mathrm{CP}$ who are not walking by 30 months and have a hip migration percentage on $x$ ray of over $30 \%$ are at high risk of hip dislocation. ${ }^{47}$ We start injections to the hip adductors and medial hamstrings from this age in children with any abnormal migration percentage (that is, over $15 \%$ ), together with 24 hour postural management. ${ }^{48}$ This incorporates the use of correct seating, sometimes with a hip spica, an abduction bed at night, and often a standing frame. ${ }^{49}$ We refer for an orthopaedic opinion if the migration percentage exceeds $30-35 \%$, at which point additional soft tissue surgery is indicated. Some children with quadriplegia sit in an uncomfortable position due to spastic hamstrings- "sacral sitting", in which case these muscles can usefully be injected. Upper limb injections are indicated mainly to aid passive movements for activities such as washing and dressing, but are rarely helpful in promoting active functioning in these children. There may be a very severe hypertonus affecting a large number of muscles in the upper and lower limbs, which is too much for focal treatment with BT-A alone. Oral antispasticity treatments can then be tried; intrathecal baclofen can be very effective in this situation, especially if there are associated spasms.

\section{THE FUTURE}

It is hoped that the effectiveness of BT-A therapy will improve in the future, partly due to changes in the injection process. The object of therapy is to incapacitate as many end plates in the target muscle as possible without causing significant spread beyond, both locally to other muscles including antagonists, and systemically to cause mild features of botulism. Critical factors are the amount of toxin given to a muscle, the volume, and the number of injections. Our study comparing 8 with 24 units/kg Dysport BT-A to the calf muscle of hemiplegics suggested that the higher dose produced a greater peak and duration of effect, but that there was an optimal dose for each individual, after which the effect was reduced due to increasing spread to antagonists. ${ }^{50}$ Baker et al also found a peak effect in diplegics, ${ }^{33} 10$ units $/ \mathrm{kg}$ to each calf being better than 5 or 15 units $/ \mathrm{kg}$. If absolute dosage is used, rather than dose $/ \mathrm{kg}$, this optimal effect is seen clearly (see fig 3). It may make more sense to use absolute number of units as a guide to dosage to a particular muscle, as motor end plates are formed before birth and are therefore independent of size. The same dose of BT-A could then be used for the muscle at all ages, varying the volume and number of injections according to individual size.

Some clinicians identify the areas of maximal density of motor end plates in a muscle using electromyography to determine the best sites for injection, ${ }^{51}$ but these may be generally distributed in most muscles, ${ }^{52}$ and the technique is impractical in children under local anaesthesia. Increasing the volume of injection is a simple way of improving efficacy, ${ }^{53}$ but excessive dilution increases spread outside the muscle. ${ }^{154}$ Dividing the dose into smaller fractions for multiple injections has been shown to improve results in treatment for blephorospasm ${ }^{55}$ and vocal dysphonia. ${ }^{56}$ This is also the case for the treatment of torticollis, ${ }^{57}$ in which the side effect of dysphonia due to local spread is also diminished, ${ }^{54}$ presumably because more of the toxin is bound better to target motor end plates with less escape. The multiple injection technique may therefore improve efficacy, without increasing spread.

Although BT-A treatment is very safe, systemic spread is a potential problem in bilateral CP, when a large amount of BT$\mathrm{A}$ is given to a number of muscles. Conventional recommendations on maximal total BT-A dose ensure systemic side effects are minimal. They do, however, restrict the maximum number of muscles which can be treated effectively at one time; for example, to only four muscle groups in the lower limb. Some clinicians have safely used a much higher total dose by dividing it between more muscles, ensuring no single muscle gets too much. ${ }^{58}$ It is possible that even higher total dosages could be tolerated by employing the multiple injection technique used in other conditions, although this will mean more use of general anaesthesia. Improving treatment along these lines depends on the accurate detection of systemic spread of toxin, such as recording changes in the electromyogram at distant muscles. ${ }^{59}$ Unfortunately this cannot be quantified, but we have found that systemic spread can be measured by its effect on the autonomic nervous system in terms of reducing vagal tone and cardiac variability ${ }^{60}$ If in future high doses of BT-A can be given safely, it would add significantly to the benefits available from this useful and fascinating natural medication.

\section{ACKNOWLEDGEMENTS}

We acknowledge all at the Derbyshire Children's Hospital involved with the Botulinum Programme, including nursing and clerical staff. 


\section{Authors' affiliations}

R E Morton, Ronnie MacKeith Child Development Centre, Derbyshire Children's' Hospital, Derby and Nottingham Queens Medical Centre and City Hospital, Nottingham, UK

J Hankinson, Physiotherapy Department, Ronnie MacKeith Child Development Centre, Derby, UK

J Nicholson, Occupational Therapy department, Ronnie MacKeith Child Development Centre, Derby, UK

Funding: REM has received research grants from Ipsen, manufacturers of Dysport BT-A.

\section{REFERENCES}

1 Scott AB. Botulinum toxin injection of eye muscles to correct strabismus. Trans Am Opthalmol Soc 1981;79:734-70.

2 Cosgrove AP, Corry IS, Graham HK. Botulinum toxin in the management of the lower limb in cerebral palsy. Dev Med Child Neurol 1994;36:386-96.

3 Koman LA, Mooney JF, Smith BP, et al. Management of cerebral palsy with botulinum toxin A: preliminary investigation. J Pediatr Orthop 1993;13:489-95.

4 Dolly JO, Lisk G, Foran PG, et al. Insights into the extended duration of neuroparalysis by botulinum neurotoxin A serotypes: differences between motor end terminals and cultured neurons. In: Brin ME, Hallett $M$, Jancovic J, eds. Scientific and therapeutic aspects of botulinum toxin. Lippincott Williams and Wilkins, 2002:91-102.

5 Brown KJ, Walsh GE. Neurology of the upper limb. In: Neville B, Goodman R, eds. Congenital hemiplegia. Clinics in Developmental Medicine, no. 150. Cambridge University Press, 2000:113-49.

6 Eames NWA, Baker R, Hill N, et al. The effect of botulinum toxin A on gastrocnemius length: magnitude and duration of response. Dev Med Child Neurol 1999;41:226-32.

7 Sackett D. Rules of evidence and clinical recommendations on the use of antithrombotic agents. Chest 1989;39(suppl 2):25-35.

8 Koman LA, Mooney JF, Smith BP, et al. Botulinum toxin type A neuromuscular blockade in the treatment of lower limb spasticity in cerebral palsy: a randomised double-blind placebo controlled trial. J Pediatr Orthop 2000;20:108-15.

9 Ubbhi T, Bhakta BB, Ives HL, et al. Randomised double-blind placebo controlled trial of the effect of botulinum toxin on walking in cerebral palsy. Arch Dis Child 2000:83:481-7.

10 Sutherland DH, Kauffmann KR, Wyatt MP, et al. Double-blind study of botulinum $A$ toxin injections into the gastrocnemius muscle in patients with cerebral palsy. Gait Posture 1999;10:1-9.

11 Love SC, Valentine JP, Blair EM, et al. The effect of botulinum toxin type A on the functional ability of the child with spastic hemiplegia; a randomised, controlled trial. Eur J Neurol $2001 ; 8$ (suppl 5):50-8.

12 Corry IS, Cosgrove AP, Duffy CM, et al. Botulinum toxin A compared with stretching casts in the treatment of spastic equines: a randomised prospective trial. J Pediatr Orthop 1998;18:304-11.

13 Flett PJ, Stern LM, Waddy $\mathrm{H}$, et al. Botulinum toxin versus fixed cast stretching for dynamic calf tightness in cerebral palsy. J Paediatr Child Health 1999;35:71-7.

14 Boyd RN, Hays RM. Current evidence for the use of botulinum toxin type A in the management of children with cerebral palsy; a systematic review. Eur J Neurol 2001;8(suppl 5):1-20.

15 Barwood S, Ballieu C, Boyd RN, et al. Analgesic effects of botulinum A; a randomised, placebo controlled clinical trial. Dev Med Child Neurol 2000:42:116-21.

16 Corry IS, Cosgrove AP, Walsh EG, et al. Botulinum toxin A in the hemiplegic upper limb: a double blind trial. Dev Med Child Neurol 1997;39:186-93.

17 Fehlings D, Rang M, Glazier J, et al. An evaluation of botulinum-A toxin injections to improve upper extremity function in children with hemiplegic cerebral palsy. J Pediatr 2000;137:331-7.

18 Fehlings D, Rang M, Glazier J, et al. Botulinum toxin type A injections in the spastic upper extremity of children with hemiplegia; child characteristics that predict a positive outcome. Eur J Neurol 2001;8(suppl 5):145-9.

19 Robertson ZC, Attfield SF, Nicholson J, et al. Kinematic and functional assessment of botulinum toxin $\mathrm{A}$ in the upper limbs of children with cerebral palsy. Submitted for publication.

20 Hurvitz EA, Conti GE, Brown SH. Changes in movement characteristics of the spastic upper extremity after botulinum toxin injection. Arch Phys Med Rehabil 2003;84:444-54.

21 Yang TF, Fu CP, Kao NT, et al. Effect of botulinum A on cerebral palsy with upper limb spasticity. Am J Phys Med Rehabil 2003;82:284-9.

22 Carr $\mathrm{L}$, Cosgrove AP, Gingras P, et al. Position paper on the use of botulinum toxin in cerebral palsy. Arch Dis Child 1998;79:271-3.

23 Forsberg $\mathrm{H}$, Tedroff $\mathrm{KB}$. Botulinum toxin treatment in cerebral palsy: intervention with poor evaluation? Dev Med Child Neurol 1997;39:635-40.

24 Cosgrove AP, Graham HK. Botulinum toxin A prevents the development of contractures in the hereditary spastic mouse. Dev Med Child Neurol 1994:36:379-85.

25 Felty $\mathbf{P}$, Morton RE, Attfield S, et al. Preliminary investigation of long term injections of botulinum toxin-A [Dysport] into calf muscles of children with hemiplegic cerebral palsy [abstract]. Dev Med Child Neurol 2001;43(suppl 90): 13-14.

26 Jancovic J, Schwartz K. Response and immunoresistance to botulinum toxin injections. Neurology 1995;45:1743-6.
27 Herrmann J, Mall V, Bigalke H, et al. Neutralising antibodies and secondary non response in treatment of cerebral palsy with botulinum neurotoxin. Scientific poster no. 30. AACPDM 54th annual meeting, 2000.

28 Bohannon RW, Smith MB. Inter-rater reliability of a modified Ashworth scale of muscle spasticity. Phys Ther 1987;67:206-7.

29 Mackay AH, Lobb GL, Walt SE, et al. The reliability and validity of the observational gait scale in children with spastic diplegia. Dev Med Child Neurol 2003:45:4-11.

30 Graham HK, Aoki KR, Ramo IA, et al. Recommendations for the use of botulinum toxin $A$ in the management of children with cerebral palsy. Gait Posture 2000;11:67-79.

31 Bourke-Taylor H. Melbourne assessment of unilateral upper limb function; construct validity and correlation with the Pediatric Evaluation of Disability Inventory. Dev Med Child Neurol 2003;45:92-6.

32 DeMatteo C, Law M, Russell D, et al. Quality of upper extremity skills test. Neurodevelopmental Clinical Research Unit, Hamilton, Ontario.

33 Baker R, Jasinski M, Maciag-Tymecka I, et al. Botulinum toxin treatment of spasticity in diplegic cerebral palsy; a randomised double-blind placebo controlled dose ranging study. Dev Med Child Neurol 2002;44:666-75.

34 RN, Graham HK. Botulinum toxin A in the management of children with cerebral palsy: indications and outcome. Eur J Neurol 1997;4(suppl 2):S15-22.

35 Morton RE, Murray- Leslie CF. The role of botulinum in the management of cerebral palsy. Curr Paediatr 2001;11:235-9.

36 Bakheit AMO, Severa S, Cosgrove A, et al. Safety profile and efficacy of botulinum toxin A (Dysport) in children with muscle spasticity. Dev Med Child Neurol 2001;43:234-8.

37 Graham HK, Aoki KR, Autti-Ramo I, et al. Recommendations for the use of botulinum toxin type A in the management of cerebral palsy. Gait Posture 2000;11:67-79.

38 Palisano R, Rosenbaum P, Walter S, et al. Gross Motor Function Classification System for cerebral palsy. Dev Med Child Health 1997;39:214-23.

39 Winters TF, Gage JR, Hicks R. Gait patterns in spastic hemiplegia in children and young adults. J Bone Joint Surg (Am) 1987;69:437-41

40 Autti-Ramo I, Larsen A, Taimo A, et al. Management of the upper limb with botulinum toxin $A$ in children with spastic type cerebral palsy and acquired brain injury; clinical implications. Eur J Neurol 2001;8(suppl 5):136-44.

41 Ward $A B$. The use of botulinum toxin type $A$ in spastic diplegia due to cerebral palsy. Eur J Neurol 1999:6(suppl 4):S95-8.

42 Westhoff B, Seller K, Wild A, et al. Ultrasound guided botulinum toxin injection technique for the ileopsoas muscle. Dev Med Child Neurol 2003;45:829-32

43 Patrick JH, Roberts AP, Cole GF. Therapeutic choices in the locomotor management of the child with cerebral palsy-more luck than judgement? Arch Dis Child 2001;85:275-9.

44 Campbell WM, Ferrel A, McLaughlin JF, et al. Long term safety and efficacy of continuous intrathecal baclofen. Dev Med Child Neurol 2002;44:660-5.

45 McLaughlin J, Bjornson K, Temkin M et al. Selective dorsal rhizotomy; metaanalysis of three randomised controlled trials. Dev Med Child Neurol 2002;44:17-25.

46 Bleck EE. Orthopaedic management in cerebral palsy. Clinics in developmental medicine 99/100. London: MacKeith Press, 1987.

47 Scrutton D, Smeeton N. Hip dysplasia in bilateral cerebral palsy; incidence and natural history in children aged 18 months to five years. Dev Med Child Neurol 2001;43:586-600

48 Boyd RN, Dobson F, Parrott J, et al. The effect of botulinum toxin type A and a variable hip abduction orthosis on gross motor function; a randomised, controlled trial. Eur J Neurol 2001;8(suppl 5):109-19.

49 Pountney T, Mandy A, Green E, et al. Management of hip dislocation with postural management. Child Care Health Dev 2002;28:179-85.

50 Polak F, Morton RE, Ward C, et al. Double blind comparison study of two doses of botulinum toxin $\mathrm{A}$ injected into calf muscles in children with hemiplegic cerebral palsy. Dev Med Child Neurol 2002;44:551-5

51 Simpson DM. Botulinum toxin in the treatment of spasticity: clinical trials and end-plate localisation. Eur J Neurol 1997;4(suppl 2):S31-2.

52 Burke RE. The structure and function of motor units. In: Karpati G Hilton-Jones D, Griggs RC, eds. Disorders of voluntary muscle, 7th edn. Cambridge University Press, 2001.

53 Kim HS, Hwang Yeong ST, Lee YT, et al. Effect of muscle activity and botulinum toxin dilution and volume on muscle paresis. Dev Med Child Neurol 2003;45:200-5.

54 Blackie JD, Lees AJ. Botulinum toxin in spasmodic torticollis. J Neurol Neurosurg Psychiatry 1990;53:640-3.

55 Borodic GE, Cozzolino D, Ferrante R, et al. Innervation zone of the orbicularis oculi muscle and implications for botulinum A therapy. Opthal Plast Reconstr Surg 1991; 7:54-60.

56 Ludlow CL, Naunton RF, Sedory SE, et al. Effects of botulinum toxin injections on speech and adductor dysphonia. Neurology 1988;38:1220-5.

57 Borodic GE, Pearce B, Smith K, et al. Botulinum A for spasmodic torticollis; multiple versus single injection points per muscle. Head and Neck 1992;14:33-7

58 Molenaers G, Desloovere K, Eyssen M, et al. Botulinum toxin type A treatment of cerebral palsy; an integrated approach. Eur J Neurol 1999;(suppl 4):S51-7.

59 Lange DJ, Brin MF, Warner CL, et al. Distant effects of local injection of botulinum toxin. Muscle Nerve 1987;10:552-5.

60 Robertshaw K, Watson L, Parkin T, et al. Autonomic side effects of treatment with botulinum toxin $A$ in children with cerebral palsy. Submitted for publication. 